\title{
A FORMAÇÃO COMO LÍDER SOCIAL NA PRODUÇÃO DE CUIDADOS EM SAÚDE. A POPULAÇÃO EM SITUAÇÃO DE RUA
}

\section{Training as a social leader in health care production. The homeless people population}

\author{
Bianca Campos Oliveira \\ Universidade Federal Fluminense \\ Margareth Santos Zanchetta \\ Daphne Cockwell School of Nursing, Ryerson University, \\ Toronto, Canada. \\ Beatriz Guitton Renaud Baptista de Oliveira \\ Universidade Federal Fluminense \\ Informações do artigo \\ Recebido em 06/11/2020 \\ Aceito em 07/06/2021 \\ doi>: https://doi.org/10.25247/2447-861X.2021.n252.p49-73
}

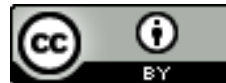

Esta obra está licenciada com uma Licença Creative Commons Atribuição 4.0 Internacional.

\section{Como ser citado (modelo ABNT)}

OLIVEIRA, Bianca Campos; ZANCHETTA, Margareth Santos; OLIVEIRA, Beatriz Guitton R. B. de. A formação como líder social na produção de cuidados em saúde. A

população em situação de rua. Cadernos do CEAS:

Revista Crítica de Humanidades. Salvador/Recife, v. 46, n. 252, p. 49-73, jan./abr. 2021. DOI: https://doi.org/10.25247/2447-861X.2021.n252.p49-73

\begin{abstract}
Resumo
Objetivo: Este relato de experiência reporta a participação dessa autora como bolsista do programa governamental Emerging Leaders in the Americas Program (ELAP), com o objetivo primeiro, de conhecer a prática de Enfermagem de rua na cidade de Toronto (Canadá) na promoção da saúde, nos cuidados diretos e da educação para o autocuidado com a população em situação de rua e usuária de drogas injetáveis assim como o trabalho de prevenção de danos em tal contexto de prática. Segundo, o de criar um modelo conceitual para guiar a futura fase de análise dos dados a serem colhidos na cidade de Niterói (estado do Rio de Janeiro). Metodologia: Abordagem descritiva, do tipo relato de experiência, que descreve a trajetória de entrada de campo de trabalho da doutoranda brasileira em locais de produção de implantação da assistência e de cuidado em saúde para a População em situação de rua na cidade de Toronto (Canadá), no período de fevereiro a junho de 2020. Conclusão: Finalmente, a oportunidade de participação no ELAP produziu múltiplos dividendos relativos a conscientização da doutoranda em seu novo papel de líder social sobre a temática, bem como influenciadora de demais enfermeiros brasileiros, estudantes de graduação e pós graduação acerca da Enfermagem como uma profissão capaz de realizar uma advocacia política e defender e assegurar os direitos das pessoas às quais prestam cuidados, em especial pessoas em situação de vulnerabilidade social. Bem como de participar ativamente na tomada de decisões, reformulações de políticas públicas e ações governamentais.

Palavras-Chave: Promoção da saúde. Enfermagem global. População em situação de rua. Drogas ilícitas injetáveis. Internacionalização da educação. Toronto.
\end{abstract}

\section{Abstract}

Objective: This experience report reports the participation of this author in the Americas Program (ELAP), with the first objective of knowing the practice of Street Nursing in the city of Toronto (Canada) in health promotion, direct care and education for self-care with the homeless population and injecting drug users as well as the work of damage prevention in this practice context. Second, to create a conceptual model to guide the future phase of data analysis to be collected in the city of Niterói (Rio de Janeiro state). Methodology: Descriptive approach, of the experience report type, describing the trajectory of the Brazilian doctoral student's entry into the field of work in sites of production of health care and assistance implementation for the homeless population in the city of Toronto (Canada), from February to June 2020. Conclusion: Finally, the opportunity to participate in ELAP produced multiple dividends related to the awareness of the doctoral student in her new role as a social leader on the theme, as well as influencer of other brazilian nurses, undergraduate and graduate students about nursing as a profession capable of performing a political advocacy and defend and ensure the rights of people to whom they provide care, especially people in situations of social vulnerability. As well as to actively participate in decision making, reformulations of public policies and governmental actions.

Keywords: Health promotion. Global nursing. Homeless population, injecting illicit drugs. Internationalization of education, Toronto. 


\section{Introdução}

O constante desafio enfrentado pelos profissionais exercendo a saúde coletiva concentra-se em tornar as instituições e serviços de saúde, responsiva às complexas demandas de assistência primária de saúde (VIEIRA, 2017). Dentre as populações vivendo extremas iniquidades em saúde, citamos a populacao em situação de rua, principalmente quando se constitui como dependentes de drogas ilícitas injetáveis (UNITED NATIONS, 2020). O consumo de tais drogas e os subsequentes danos provocados à saúde desta população requer dos serviços de saúde, ações curativas e de promoção de saúde de forma integrada, envolvendo diversos setores da sociedade e da administração pública (REZAEl, 2017).

Atualmente, esta situação constitui um problema global de saúde pública (WORLD HEALTH ORGANIZATION, 2018), em especial para a Enfermagem global (NARASIMHAN, 2019; FOWLER, 2020) pois testa a factibilidade de conhecidos modelos conceituais/teóricos de promoção de saúde populacional (e.g. modelo de redução de danos (PAULA; 2020), enquanto propicia a emergência de novos modelos e estratégias logicamente mais responsivos aos determinantes sociais de saúde, como fatores sociais, econômicos, culturais, étnicos/raciais, psicológicos e comportamentais (HEALTH CANADA, 2019; FIORATI, 2016). Cumpre-nos destacar a experiência canadense no enfrentamento deste problema de saúde pública pautado em sua conhecida liderança na área de promoção da saúde (HASFORD, 2019; RAMSAY, 2019; POTTIE; 2020). A concepção de programas a esta população tenta coibir os efeitos de políticas econômicas, os efeitos do crescente adoecimento emocional e mental, e ao multidimensional sofrimento dos indivíduos (PANKRATZ, 2017; PANKRATZ, 2018; WORTON, 2018, HARRIS, 2019)

Priorizando a expansão do conhecimento sobre a prática de enfermeira(o)s atuando com tal população em um contexto consolidado na perspectiva de promoção de saúde populacional, a autora principal deste artigo, enfermeira doutoranda brasileira, com experiência na área da atenção primária à saúde, explorou tal conhecimento na cidade de Toronto-Canadá. Enquadrando na sua experiência nas áreas de Gestão Pública, Saúde Pública, Políticas Públicas e Sociais e Saúde Coletiva, seu objeto de pesquisa no doutoramento explora o tratamento de feridas em condições crônicas e em sua avaliação no âmbito da saúde pública com a população em situação de rua e a integridade da pele, uma área de grandes lacunas de conhecimento no Brasil.

Este relato de experiência reporta a participação dessa autora como bolsista do programa governamental Emerging Leaders in the Americas Program (ELAP) gerenciado pelo Global Affairs Canada (GOVERNMENT OF CANADA, 2020). O ELAP criado em 2009 pelo Governo do Canadá oferece aos estudantes de graduação e pos-graduacao stricto sensu, oriundos da América Latina e do Caribe, oportunidades de treinamento em pesquisa ou estudos e apoia o desenvolvimento de capital 
humano e a formação de líderes emergentes nas Américas capazes de discutir criticamente questões voltadas à governança democrática e responsável, problemas sociais, direitos humanos, meio ambiente, diversidade e povos indígenas (GOVERNMENT OF CANADA, 2020).

\section{- Contexto acadêmico da experiência}

A período do treinamento em pesquisa deu-se na cidade de Toronto cuja imersão cultural deu-se na Daphne Cockwell School of Nursing (DCSON), Faculty of Community Services-Ryerson University e em várias instituições e organizações comunitárias que foram visitadas na fase de entrada de campo em locais de produção de cuidado em saúde para a população em situação de rua. Esta entrada de campo permitiu identificar, analisar e compreender ações integrativas de promoção da saúde destinados a esta população. Isto feito dentro de um contexto de internacionalização de pesquisa e aprendizado em países que vivenciam a mesma problemática, a fim de viabilizar experiências e conhecimentos sobre tal questão, enriquecendo as pesquisas e gerando um retorno social e político ao país de origem.

Tal treinamento em pesquisa intensivo e desafiador teve dois objetivos. Primeiro, o de conhecer a prática de Enfermagem de rua na cidade de Toronto (Canadá) na promoção da saúde, nos cuidados diretos, e, da educação para o autocuidado com a população em situação de rua e usuária de drogas injetáveis, assim como o trabalho de prevenção de danos em tal contexto de prática. Segundo, o de criar um modelo conceitual para guiar a futura fase de análise dos dados a serem colhidos na cidade de Niterói (estado do Rio de Janeiro).

Esse processo de internacionalização, de exploração de evidências com peritos da prática, e, de entrada e imersão de campo em locais de produção de cuidados em saúde para tal população, foi mediado pela supervisora canadense, docente da diáspora brasileira, com experiência em design de modelos conceituais, letramento em saúde, autocuidado e atenção primária à saúde com população socialmente vulnerável. Foi seu papel de intermediação entre culturas de Enfermagem nas questões de cuidados primários de saúde no Canadá e no Brasil que possibilitou o acesso às informações disponibilizadas no site do Registered Nurses Association of Ontario (RNAO) para aprender sobre Best Practice Guidelines-BPGs (Diretrizes de Boas Práticas), que oferecem uma abordagem clínica sistemática para feridas crônicas, também entre os mais socialmente vulneráveis (Registered Nurses' Association of Ontario). A outra colaboração ímpar (apesar de ter sido não-oficial) e extremamente relevante foi estabelecida com um enfermeiro lotado no Toronto Public Health (TPH), e com uma enfermeira que listou as possíveis organizações para a busca de colaboração e para visitas técnicas. A mesma facilitou visitas técnicas a organizações comunitárias, e contatos com enfermeira(o)s que atuam diretamente nas Street Nurse Clinics (Clínicas de Enfermagem de Rua) possibilitando 
compreender as intervenções de cuidados de enfermagem nas ruas, bem como ensinamentos para o autocuidado.

Finalmente, o processo de criação do modelo conceitual, resultado final almejado neste treinamento de pesquisa foi objetivo da colaboração intelectual com duas outras enfermeiras pesquisadoras na área de saúde comunitária lotadas na DCSON e na Lawrence S Bloomberg Faculty of Nursing (University of Toronto) esta última, também membro da diáspora brasileira.

\section{- Contexto social da experiência}

Ontário é a maior província do Canadá, com mais de 13,2 milhões de residentes em 2010, somada a população transitória composta por trabalhadores temporários e estudantes, todos com acesso a seguro de saúde com financiamento público para serviços médicos e hospitalares (MORIN, 2017). A cidade de Toronto localizada na província de Ontário é o cenário do campo de exploração da prática e da conceitualização dessa assistência diferenciada. Toronto é a maior cidade do Canadá cujo último levantamento realizado sobre a população em situação de rua em Toronto foi realizado em 2018 pela quarta avaliação de necessidades de rua que realiza a contagem pontual de pessoas que enfrentam falta de moradia (TORONTO PUBLIC HEALTH; 2018). Sua população geral é constituída de cerca de 2.9 milhões de habitantes, sendo 51.2\% da população composta de imigrantes e aproximadamente 8.715 pessoas estão vivendo em situação de rua em Toronto e mais de 17.000 indivíduos usam abrigos para homeless, sem-teto, na cidade a cada ano, um número expressivo quando comparado aos números do próprio país, onde estima-se que 35.000 canadenses vivem nas ruas e mais de 235.000 pessoas estarão nas ruas ao longo de um ano. Desta forma, embora o Canadá seja uma das dez principais nações comerciais da economia global, a população em situação de rua continua sendo um problema social significativo. (TORONTO PUBLIC HEALTH; 2018; LATIMER; 2017).

Cabe ressaltar a existência de uma população de refugiados que recentemente em 2019 atravessaram as fronteiras, segundo dados da Immigration, Refugees and Citizenship Canada (Imigração, Refugiados e Cidadania do Canadá) e da Canada Border Services Agency (Agência de Serviços de Fronteira do Canadá). Em 2019 cerca de 64.050 indivíduos- realizaram uma solicitação de asilo no Canadá. Destes cerca de 26.880 se encontram na província de Ontário (Government of (Canada). Assim essa população é direcionada aos abrigos existentes no Canadá que em muitas das vezes não conseguem abarcar a população de refugiados e a população em situação de rua, agravando assim o problema de pessoas sem abrigo no país.

Na população total estimada neste último levantamento realizado foi de 8715 pessoas em situação de rua, $94 \%$ estavam em locais fechados como abrigos e $6 \%$ (533) vivem nas ruas. Do total 
da população em situação de rua, cerca de $38 \%$ são identificados como indígenas, $11 \%$ como LGBTQ2S (Lésbicas, Gays, Bissexuais, Transgêneros, Transsexuais, Queer, Questionadores e Espiritualistas), 10\% eram idosos (6o anos ou mais) e 10\% eram jovens ( 16 a 24 anos) (TORONTO PUBLIC HEALTH; 2018). Essa população têm uma expectativa de vida de apenas 42-52 anos e sua taxa de mortalidade é de 3-4 vezes maior que a média nacional (HENRY, 2017). No âmbito da saúde mental, cerca de $50 \%$ a $57 \%$ desta população apresentavam uma doença mental diagnosticável, sendo que cerca de $80 \%$ destas doenças não são tratadas. Além de problemas de saúde mental, $62 \%$ têm dependência de álcool e 58\% de outras drogas (HENRY, 2017).

O uso de álcool, tabaco e substâncias ilícitas são importantes contribuintes para a carga global de morbimortalidade, em específico da população em situação de rua. O tabaco e o álcool são usados com mais frequência e dão maiores contribuições ao ônus da doença que as drogas ilícitas; sabemos que o ônus deste último (álcool) apresenta maiores problemas de subestimação devido a maiores limitações da disponibilidade e qualidade dos dados (PEACOCK, 2018).

Um dos grandes problemas de saúde pública enfrentados pelo Canadá e principalmente na província de Ontário, e em especial em Toronto é o transtorno por uso de opióides. Em um último levantamento do número de indivíduos que procuram tratamento para sua dependência de opióides notou-se um aumento do número de inscrições para o tratamento de 6.000 para mais de 40.000 no período de 2000 a 2016 em Ontário (ONTARIO DRUG POLICY RESEARCH NETWORK, 2016; MORIN, 2017).

A epidemia de opióides é uma questão complexa e multifatorial, de acordo com um estudo de coorte retrospectivo que utilizou dados administrativos, $87 \%$ dos indivíduos com transtorno do uso de opióides em Ontário também têm um distúrbio diagnosticado de saúde mental, sendo mais prevalente em indivíduos na faixa etária de 15 a 34 anos. Foram evidenciados que ações voltadas para os determinantes sociais de saúde tem um papel importante na prevenção e tratamento deste (Morin, 2017; Matsumoto, 2017).

A realidade social e de saúde acerca da populacao em situação de rua brasileira não indica ser muito diferente da descrita acima, já que dados apresentados em um levantamento governamental sobre esta população em 2008 identificou 31.922 pessoas maiores de 18 anos vivendo em situação de rua (MINISTÉRIO DO DESENVOLVIMENTO SOCIAL E DO COMBATE À FOME, 2008; TILIO et al., 2016; ENGSTROM et al., 2016).

Sobre suas condições de saúde, 29,7\% dos entrevistados afirmaram ter algum problema de saúde que necessita de cuidados contínuos. Os problemas mais citados foram: hipertensão $(10,1 \%)$, problema psiquiátrico/mental $(6,1 \%)$, HIV/aids $(5,1 \%)$ e problemas de visão/cegueira $(4,6 \%)$. Cerca de $18,7 \%$ faziam uso de algum medicamento e destes, $48,6 \%$ afirmaram que a principal via de acesso aos 
medicamentos é a Atenção Básica $(A B)$ e que quando ficavam doentes, procuravam o hospital/emergência em primeiro lugar (BRASIL, 2008).

Segundo esta mesma pesquisa realizada em 2008, os principais motivos pelos quais essas pessoas passaram a viver e morar na rua se referem aos problemas de alcoolismo e/ou drogas (35,5\%), ao desemprego (29,8\%) e (29,1\%) às desavenças com familiares (BRASIL, 2008). A justificativa da ida e permanência nas ruas devido a uma escolha pessoal também aparece, não de forma frequente e expressiva, porém deve ser levada em consideração. Ao relatarem a escolha pela vida nas ruas, justificam que a busca pela sensação de liberdade é a principal motivação (ROSA, 2019).

Mais recentemente, em 2015, o Instituto de Pesquisa Econômica Aplicada (IPEA) realizou uma estimativa 101.854 pessoas em situação de rua no Brasil, levando em consideração variáveis como crescimento demográfico, dinamismo urbano, vulnerabilidade social e serviços voltados à população de rua, bem como o número de pessoas em situação de rua cadastradas no Cadastro Único para Programas Sociais do governo federal (Cadastro Único). O IPEA ressalta que há limitações, como a impossibilidade de informar estimativas precisas para cada município, necessitando assim da realização de pesquisas municipais e a incorporação desta população incorporada ao Censo de 2020 (agora previsto para 2022) (NATALINO, 2016).

O Instituto Brasileiro de Geografia e Estatística (IBGE) declarou que para a incorporação da população em situação de rua, seria necessário a mudança de toda a metodologia do Censo. Assim cabe salientar que é necessário que se reconheça o perfil desta população em situação de vulnerabilidade, a fim de subsidiar a tomada de decisões, bem como a criação de novas políticas públicas ou aperfeiçoamento das já existentes. Políticas tais que ainda são recentes e seus impactos consolidados estão por ser documentados.

Em Toronto existe uma vasta rede de organizações assistindo tal população tais como Central Toronto Community Health Centres, All Saints Church - Community Centre, The Scarborough Hospital Addiction Program, mas a agência líder pertencente ao governo provincial chama-se Toronto Public Health (TORONTO PUBLIC HEALTH, 2020). TPH foi criado em 1883 e se concentra na proteção e promoção da saúde dos residentes na área geográfica denominada Greater Toronto Area (GTA). Referente a população em situação de rua, a estratégia de promoção da saúde se apoia na filosofia de redução de danos e relações participativas, enfatizando quando e como a assistência integral e os cuidados são prestados. Além do desenvolvimento de parcerias com organizações sociais como, abrigos, organizações não governamentais (ONGs) e grupos religiosos (TORONTO;2020).

Em Toronto existem 9 locais de injeção segura (Fred Victor; Moss Park Overdose Prevention Site; Parkdale Queen West Community Health Centre; Parkdale Queen Community Health Centre; Regent Park Community Health Centre; South Riverdale Community Health Centre KeepSIX; Street Health; St. Stephen's Community House Corner Drop In Centre; Toronto Public Health The Works). O 
objetivo geral da assistência prestada nesses locais é oferecer um ambiente seguro e higiênico para que os indivíduos injetem-se com drogas pré-obtidas por eles sob a supervisão de pessoal qualificado (TORONTO PUBLIC HEALTH, 2020). Além da injeção supervisionada, os indivíduos que utilizam esses serviços de saúde recebem suprimentos estéreis de injeção, e quando solicitado, educação sobre prevenção e intervenção em overdose. Cabe ressaltar que na dinâmica desses serviços e nas formas de registro existe a total proteção da identidade da clientela com absoluto uso do anonimato, a fim de manter o sigilo dos mesmos. Ainda são disponíveis serviços de aconselhamento em saúde e encaminhamentos para tratamento de drogas, moradia, apoio à renda e outros serviços. Esses importantes serviços de saúde fazem parte do Toronto's Overdose Action Plan, que foi adotado pelo Board of Health em março de 2017 (TORONTO PUBLIC HEALTH, 2020).

\section{Breve apresentação sobre estado de conhecimento sobre o tema}

A abordagem de redução de riscos promovendo a saúde dos usuários de drogas injetáveis no Canadá

O objetivo dos locais de injeção segura é reduzir a morbimortalidade entre pessoas dependentes de drogas e que usam drogas em espaços públicos, e reduzir o uso visível de drogas e problemas afins em espaços públicos (FORTSON, 2018). Essas instalações visam:

a) proporcionar um ambiente mais seguro e estéril para o consumo de uma droga pré-obtida; reduzir a transmissão de vários vírus transmitidos pelo sangue, como hepatite C e HIV;

b) reduzir a incidência de overdoses e mortes relacionadas a medicamentos; gerenciar os efeitos do uso de drogas; fornecer educação em saúde e incentivar os usuários a se envolverem com serviços relevantes, primeiro passo no processo de tratamento;

c) facilitar outros apoios e referências sociais, médicas e de tratamento (FORTSON, 2018).

Há também benefícios sociais e públicos mais amplos que inclui:

a) atrair usuários para longe de configurações de consumo "não autorizadas" (como galerias, becos, banheiros públicos) para um ambiente mais seguro e limpo; redução de injeção pública / de rua;

b) reduzir a incidência de "lixo derivado do uso de drogas" como seringas;

c) permitir que as agências possam monitorar os mercados de drogas (por exemplo, o surgimento de novas drogas), reduzir o custo de responder a danos e mortes 
relacionados a drogas e reduzir o índice de criminalidade através do encaminhamento destes indivíduos para o tratamento e reinserção social (FORTSON, 2018).

Essa abordagem aumentou em sua aceitação à medida que as instalações de injeção supervisionadas foram criadas em países como Alemanha, Suiça, Espanha, Holanda, Austrália, Estados Unidos da América e Canadá. De fato, tem existido um interesse crescente no uso de instalações de injeção supervisionada para combater os problemas sociais e de saúde pública como criminalidade, problemas de saúde, descarte inadequado de seringas e outros lixos oriundos do uso de drogas, representando um risco significativo para a saúde de indivíduos que os utilizam e para a coletividade, devido a contaminação ambiental com material biológico (RUDD, 2016; MYER, 2018). Pesquisas internacionais mostram que os serviços supervisionados de injeção reduzem as overdoses ocasionados por drogas, salvam vidas e limitam a disseminação do HIV e hepatite C relacionados a práticas de injeção inseguras, diminuem a criminalidade. (GADDIS, 2017; MYER, 2018; Bergamo, 2020).

Dados observacionais de pesquisadores e residentes que moram perto de instalações de injeção supervisionadas revelam resultados interessantes. Há uma diminuição na injeção pública de drogas, menos seringas descartadas de maneira insegura e menor frequência de queixas sobre incômodos pela injeção pública de drogas. Ainda que a implantação de instalações supervisionadas de injeção nas localidades, não aumentaram a quantidade de uso de drogas injetáveis na região ou criaram novos usuários de drogas injetáveis (POTIER, 2014)

Desta forma as instalações de injeção supervisionadas visam melhorar a ordem pública, reduzindo a atividade e o uso público de drogas. Também acontece uma melhora da saúde e o bemestar geral dos usuários de drogas na comunidade, aumentando o acesso destes a serviços e opções de tratamento. Como resultado reduz-se o impacto negativo que o uso de drogas tem sobre a comunidade e melhora a segurança geral, com redução significativa dos índices de violência e os crimes relacionados às drogas (MYER, 2018; IRWIN, 2017).

Além da literatura científica, pode-se aprender com a experiência e a expertise canadense com iniciativas comunitárias pelos serviços de saúde pública e pela política, bem como ações implantadas por voluntários quer sejam eles, enfermeira(o)s, assistentes sociais, médica(o)s, psicóloga(o)s, farmacêutica(o)s e estudantes, e ainda, membros da sociedade civil. Tal trabalho intersetorial está amplamente disseminado e acessível ao público geral retratando a situação da epidemia do uso dos opióides nas ruas, principalmente das grandes cidades tais como Vancouver, Toronto, Montreal. Informação que também se encontra disponível em páginas Facebook dos grupos em ativismo político, Twitter, Instagram tais como: 


\begin{tabular}{|l|l|}
\hline \multicolumn{1}{|c|}{ Midías Sociais } & \multicolumn{1}{c|}{ Links } \\
\hline Street Nurses Network & https://www.facebook.com/groups/StreetNursesNetwork/ \\
\hline Street Health & https://www.facebook.com/StreetHealthTO/?ref=br_rs \\
\hline Nursing Students for Social Justice - Ryerson & https://www.facebook.com/groups/ryersonnssj/?ref=search \\
\hline Toronto Drup-in Network & https://www.facebook.com/TorontoDropinNetwork/ \\
\hline CBC News & https://www.facebook.com/cbcnews/ \\
\hline Streets Of Toronto & https://www.facebook.com/StreetsOfToronto/ \\
\hline All Saints Toronto & https://www.instagram.com/p/B6aggmlhXxG/ \\
\hline Rise Up Movement & https://www.instagram.com/rise.up.movement/ \\
\hline
\end{tabular}

Tais mídias sociais militam pela solução de causas destrutivas dos determinantes sociais da saúde dessa população vulnerável. Tais como, o acesso a moradia, a alimentação, aos serviços de saúde e aos cuidados de saúde em eventos de risco de vida iminente. Esta mobilização da sociedade civil representa ações interdisciplinares e intersetoriais compatíveis com o Population Health Promotion Model (PHPM) (Modelo Canadense de Promoção da Saúde da População) (Hamilton \& Batti) que revelam a liderança cidadã em defesa dos mais vulneráveis ao exigir e monitorar as ações dos governos canadenses provinciais e federal. Um exemplo de empoderamento coletivo a ser replicado por países que enfrentam epidemia de consumo de drogas nas ruas.

Por outro lado, existem posições sociais e científicas contrárias a tal abordagem, argumentando que a mesma normaliza o uso de drogas e que não há evidências científicas que comprovem melhorar a adesão ao tratamento e a recuperação de pessoas em uso de drogas. E que desta forma os orçamentos destinados à saúde pública deveriam ser investidos em outros serviços para recuperação e tratamento de indivíduos em uso de drogas (KNOPF, 2018).

\section{Marco conceitual}

O treinamento de pesquisa em sua ampla perspectiva conceitual e seu trabalho de campo correlato, foi conceitualmente enquadrado pelo modelo canadense de promoção de saúde populacional (Hamilton \& Batti). O Population Health Promotion Model (PHPM) que inclui entre as dimensões da promoção da saúde, os determinantes sociais de saúde (por exemplo, redes de apoio social, práticas pessoais de saúde, habilidades de enfrentamento e acesso a serviços de saúde), 
relacionados a locais e condições em que os indivíduos nascem, vivem, trabalham e se desenvolvem (CANADIAN COUNCIL ON SOCIAL DETERMINANTS OF HEALTH; 2015).

O modelo indica 5 ações integrativas de importância capital para promoção da saúde otimizando os determinantes sociais de saúde que incluem:
a) fortalecer a ação da comunidade;
b) criar ambientes de apoio;
c) desenvolver habilidades pessoais;
d) construir um público saudável;
e) reorientar os sistemas de saúde.

Tais ações devem ser implementadas de modo integrado em cada iniciativa de promoção de saúde. O modelo também indica níveis de intervenções para promover a saúde do indivíduo, do núcleo familiar e da sociedade no seu âmbito geral. A adequação deste modelo para guiar este treinamento em pesquisa deve-se à sua capacidade de apoiar o entendimento de como abordar o reconhecimento inicial de iniquidades em saúde vivenciadas pela população em situação de rua (por exemplo, exclusão social, risco de overdose, agressão física, suicídio, envolvimento com crimes e prisão). Essas bases conceituais também estão presentes na abordagem de redução de danos (STAMLER, 2008; HEALTH CANADA, 2018)

Na prática da Enfermagem comunitária canadense a abordagem da promoção da saúde está também a redução de danos (YOUNG, 2017), assim como preconizada pelo Health Canada (HEALTH CANADA, 2018). Sendo definida como uma abordagem abrangente, compassiva e colaborativa da saúde pública ao transtorno do uso de substâncias, com o intuito de reduzir os efeitos nocivos, sociais, econômicos e de saúde no indivíduo, família ou comunidade, e tendo como objetivo de reduzir riscos, melhorar a saúde e conectar os indivíduos com outros serviços sociais e de saúde essenciais. Já a abordagem da redução de danos aplicável ao uso de drogas ilícitas é definida pela International Harm Reduction Association, (Associação Internacional de Redução de Danos) como sendo um conjunto de políticas e práticas que objetiva reduzir os danos associados ao uso de drogas psicoativas, visando primeiramente reduzir as consequências adversas para a saúde, sociais e econômicas do uso de drogas lícitas e ilícitas, sem necessariamente reduzir o seu consumo. Assim a redução de danos visa à prevenção aos danos decorrentes do uso de dorgas, ao invés da prevenção de seu uso (GOMES et al., 2018; SOUZA et al., 2018; HARM REDUCTION INTERNATIONAL, 2020).

As intervenções voltadas para a redução de danos são pragmáticas, possíveis, efetivas, seguras e custo efetivas, assim são desenvolvidas intervenções com compromisso de basear suas políticas e práticas na mais forte evidência científica existente. Desta forma maior parte das ações de redução de danos podem ser de baixo custo, fáceis de implementar e podem ter um alto impacto na 
saúde individual e comunitária, em países como o Brasil onde os recursos financeiros voltados para a saúde pública são escassos, os benefícios seriam maiores na escolha de medidas de baixo custo/alto impacto ao invés de alto custo/ baixo impacto (CAMARGO, 2016; GOMES et al., 2018).

\section{Produção Científica da Enfermagem Canadense acerca da temática}

Os antecedentes históricos da Enfermagem voltada para a população em situação de rua

A "Enfermagem de rua", ou a assistência de Enfermagem a pessoas sem-teto, tornou-se uma especialidade de Enfermagem estabelecida não apenas no Canadá, mas também nos Estados Unidos, Grã-Bretanha, Europa, Austrália e nos últimos anos vem sendo desenvolvida no Brasil. Ela ocupa seu lugar na história da Enfermagem em meio a um legado de "cuidar dos pobres" ou seja de pessoas em estado de extrema vulnerabilidade social. Embora a sua história não seja amplamente documentada, Vancouver, British Columbia, aparece como o seu berço da Enfermagem de rua no Canadá. Nas narrativas de enfermeiras que atualmente prestam cuidados para a populacao em situação de rua em Vancouver, indicam que a pioneira foi a Enfermeira Grace Donald (HARDILL, 2007). Já em 1946, quando os antibióticos começaram a ser utilizados, a Enfermeira Donald forneceu tratamento para doenças venéreas e rastreamento de contatos. Ela começou a perceber que pessoas marginalizadas não estavam frequentando as clínicas e hospitais, e que grande parte em situação de vulnerabilidade atuava como profissionais do sexo, que nesta época era controlado por uma lei denominada "lei da vadiagem". Como resultado, a grande parte destas pessoas eram presas e desta forma as ações de saúde da Enfermeira Donald começaram a ser desenvolvidas principalmente em prisões, hotéis, e locais onde atuavam esses/essas profissionais do sexo (HARDILL, 2007; FOTH, 2018).

O final das décadas de 1960 e 1970 em Toronto também testemunhou o início de sensibilização para jovens transitórios que vivem em prédios abandonados que eram considerados de risco para problemas de saúde como resultado de sua falta de moradia e uso de drogas. Ações desenvolvidas nesta época buscavam principalmente auxiliar esses jovens a lidar com os problemas das drogas e os tratamentos de infecções sexualmente transmissíveis. Apesar do início do desenvolvimento destas ações por Enfermeiros, este momento não foi reconhecido como "enfermagem de rua" no Canadá, sendo reconhecido apenas em 1985, com a criação de uma organização chamada Street Health por um grupo de pessoas anteriormente sem-teto e aliados da comunidade, incluindo a Enfermeira Dilin Baker. Desta forma a primeira clínica de Enfermagem foi aberta em janeiro de 1986 em um grande centro de atendimento de Toronto usando Enfermeira(o)s voluntárias. Em 1989, a Street Health se tornou a primeira organização autônoma de Enfermagem de Ontário, financiada pelo Ministério da Saúde da província (HARDILL, 2007). 
Nos dias atuais existem enfermeira(o)s de rua trabalhando na maioria das grandes cidades canadenses de Vancouver a Halifax, além da área rural da Colúmbia Britânica e cidades menores, como Sudbury em Ontário. Podemos notar que o crescimento exponencial da Enfermagem de rua é na verdade um reflexo das respostas governamentais a um problema de social que continua a existir e apresenta um crescimento exponencial que é o aumento da pobreza e dos sem-teto em todo o país (HARDILL, 2007; FOTH, 2018).

Assim muito se evoluiu até os dias atuais, e é mais do que correto honrar a história da Enfermagem de rua no Canadá, visto que a mesma nos dias atuais se tornou uma referência para outros países por seu caráter ético, com formação técnico/científica robusta e regulamentada, e por desenvolver ações e medidas de apoio à população de rua. Porém cabe ressaltar que tais enfermeira(o)s continuam lidando com muitos dos mesmos problemas sociais e de saúde enfrentados no passado, tais como as doenças infecciosas, o uso abusivo de drogas e as mortes prematuras dessa população (HARDILL, 2007; VILLENEUVE, 2019).

Trabalhando as relações interpessoais com pessoas em situação de rua

O acesso aos serviços de saúde para pessoas marginalizadas devido à falta de moradia e uso de substâncias é facilitado pela reconstrução da confiança. Os enfermeira(o)s consideram que a interação com cada cliente é uma oportunidade de construir confiança que pode facilitar o acesso à saúde e serviços auxiliares necessário a essa população (JAMES; 2017). A falta de confiança pode afetar negativamente o acesso aos cuidados de saúde, levando a um afastamento desta população. A confiança é mais frequentemente compreendida no nível interpessoal e reflete uma preocupação moral pelo outro (JAMES, 2017). Reconstruir a confiança interpessoal entre enfermeiros e clientes é o primeiro elo na construção de uma cadeia de confiança que pode facilitar o acesso aos serviços sociais e de saúde necessários e estabelecer o vínculo necessário para o enfrentamento dos desafios complexos de saúde e assistência social que essa população apresenta (DOBERSTEIN, 2016; PAULY, 2014).

A importância das políticas organizacionais voltadas para as pessoas em situação de rua

As políticas organizacionais que apoiam a redução dos danos que essa população enfrenta, permite que os enfermeiros encontrem as pessoas 'onde estejam' e 'em seu próprio território' e contribuam para o desenvolvimento da confiança e a criação do vínculo (PAULY, 2014). A confiança entre provedores de serviços promoveu o acesso a uma gama mais ampla de serviços sociais e de saúde. A interrupção das cadeias negativas de julgamento e a construção da confiança entre agências 
fomentam o acesso desta população aos serviços, e ao mesmo tempo destaca as lacunas pré existentes e falta de vínculos sistêmicos muitas vezes ainda existentes (DOBERSTEIN, 2016).

Práticas baseadas em evidências voltadas para a população em situação de rua

A prática baseada em evidências (PBE) é definida como o uso consciente, explícito e criterioso das melhores evidências atuais na tomada de decisões sobre o atendimento de uma clientela. No âmbito de serviços voltados para a população em situação de rua, a promoção e o uso de práticas baseadas em evidências continuam a crescer. A Prática Baseada em Evidência Dentre tais PBE voltada para essa população no Canadá são: o Assertive Community Treatment, Critical Time Intervention e o Housing First.

O Assertive Community Treatment é um modelo baseado em equipe, projetado para fornecer tratamento psiquiátrico abrangente, baseado na comunidade, reabilitação e apoio a pessoas com doenças mentais graves. Um estudo realizado em 2001, descreveu o Assertive Community Treatment e analisou a evidência de 25 ensaios clínicos randomizados. A análise demonstrou que o programa é eficaz na redução da hospitalização, apresenta menor custo que o atendimento tradicional e é mais satisfatório para os clientes e seus familiares do que o atendimento padrão (PHILLIPS, 2001).

A Critical Time Intervention é uma prática baseada em evidências com tempo limitado que mobiliza apoio aos indivíduos mais vulneráveis da sociedade durante os períodos de transição. Facilita a integração da comunidade e a continuidade de cuidados, garantindo que um indivíduo tenha laços duradouros com a comunidade e sistemas de apoio durante esses períodos críticos, sendo destinados a pessoas com doenças mentais, pessoas que ficaram desabrigadas ou presas e muitos outros grupos. Estudo clínicos demonstram que a implementação da Critical Time Intervention apresentaram como resultado: uma redução da falta de moradia em três vezes, e o efeito persistiu após o final da intervenção ativa (SUSSER et al 1997); um estudo de análise econômica demonstrou que a Critical Time Intervention foi custo-efetiva (JONES et al 2003); uma redução do risco de falta de moradia durante o período de acompanhamento (LENNON et al 2005) e um estudo com 72 homens mostraram que tal intervenção estava associada a uma diminuição estatisticamente significativa nos sintomas negativos apresentados por essa população (HERMAN et al 2000).

O Housing First é uma abordagem para abrigar pessoas sem-teto que têm doenças mentais graves e distúrbios concomitantes de uso de substâncias, que prioriza o fornecimento de moradia permanente para pessoas que vivem sem-teto, terminando assim o desabrigo e servindo como uma plataforma a partir da qual eles podem buscar objetivos pessoais e melhorar sua qualidade de vida. Resultados de 4 ensaios clínicos que demonstraram que o programa Housing First aumenta a estabilidade na habitação para os clientes atendidos, são econômicos em comparação com os 
serviços tradicionais e, aumentam a utilização e adesão do cliente por outros serviços de suporte (JULIA R., 2016).

\section{Metodologia}

Abordagem descritiva, do tipo relato de experiência, que descreve a trajetória de entrada de campo de trabalho da doutoranda brasileira em locais de produção de implantação da assistência e de cuidado em saúde para a População em situação de rua na cidade de Toronto (Canadá). A experiência relatada de entrada de campo de trabalho planejada e adaptada ocorreu no período de fevereiro a junho de 2020.

Durante esse período, a supervisora canadense ofereceu suporte acadêmico que permitiu: (a) atualizar conhecimentos sobre o (i) modelo de Promoção da Saúde Populacional incluindo a abordagem de redução de danos; (ii) revisar literatura internacional sobre Enfermagem de rua e científica e profissional canadense (iii) revisar estratégias para abordar os principais determinantes sociais comprometidos da saúde da população em situação de rua (por exemplo, abrigo, moradia, acesso a serviços de saúde, educação em saúde); (b) guiar o aprimoramento do projeto de pesquisa principalmente na expansão de conhecimentos nas seguintes áreas: (i) na condução de uma revisão da literatura sobre as Diretrizes, políticas e iniciativas canadenses de Boas Práticas de Enfermagem para combater a complicação das condições de saúde nas ruas; (ii) em visitas técnicas e contato com enfermeiros lotados em Street Nurse Clinics para aprender sobre cuidados de enfermagem e filosofia e abordagem de trabalho; bem como, com Toronto Public Health para consultar os enfermeiros responsáveis pelo atendimento aos usuários de drogas nas ruas; (iii) participar das atividades online e presenciais do grupo Ryerson Nursing Students for Social Justice para aprender sobre advocacia de enfermagem para clientes socialmente vulneráveis; (c) oferecer aconselhamento e recomendação para o desenho e desenvolvimento de uma estrutura conceitual; (d) supervisionar a preparação de resumos para submissão a conferências científicas e para a redação de manuscritos científicos em parceria com a orientadora brasileira da doutoranda.

\section{- Fases do trabalho de campo}

A fim de tornar os dados desta experiência mais compreensiveis ao leitor, as fases de trabalho de campo foram dívididas em três fases: Revisão de marcos conceituais e evidências empíricas e políticas; Entrada no campo, apresentações de instituições visitadas e vivências; Modificações do plano de trabalho. 
Primeira fase: Revisão de marcos conceituais e evidências empíricas e políticas

Para a compreensão sobre a população em situação de rua em Toronto e posteriormente subsidiar o processo de imersão nos locais de cuidado em saúde para essa população. Realizou-se uma análise crítica da literatura acadêmica internacional sobre população em situação de rua. Quanto a literatura cinzenta realizou-se a leitura de materiais encontrados em sites como o Canadian Public Health Association; Government of Canada; Toronto Public Health; Sherbourne Health; All Saints Church Community Centre; All Saints Church-Community Centre; Fred Victor; The Canadian Encyclopedia; Ryerson University; além de capítulos de livro, livros, protocolos que abordam temas das políticas públicas de saúde, políticas sociais e políticas econômicas, voltadas à população em situação de rua, assim como problemas globais existentes diante deste cenário e os modelos e estratégias logicamente mais responsivos aos determinantes sociais de saúde voltados a essa população. Os mesmos temas guiaram a revisão da literatura científica.

Segunda fase: Entrada no campo, apresentações de instituições visitadas e vivências

Nesta segunda fase foi realizada a inserção nos serviços de assistência a população de interesse, assim como participação em eventos locais sobre a temática, bem como reuniões com enfermeira(o)s que atuam na assistência a essa população a fim de obter uma troca de experiências e de compreender o contexto do cotidiano dessa população.

Foram realizadas visitas ao centro comunitário All Saints Community Centre-Church Toronto durante 12 horas. A All Saints Community Centre-Church Toronto é uma paróquia anglicana criada em 1972, tem seus princípios baseados na justiça social, atuando para proteger os direitos, a saúde e a segurança de indivíduos em vulnerabilidade (ALL SAINTS, 2020). Dentre os projetos desenvolvidos destaca-se o All Saints Drop-in onde são oferecidos serviços como: nutrição e culinária, acesso ao computador, jogos de tabuleiro, atividades sociais de lazer; consultas de saúde e bem-estar com uma enfermeira; e ainda suprimentos para redução de danos e troca de agulhas (ALL SAINTS, 2020). Essa informou que o Centro comunitário, fornece serviços de assistência de saúde básicos e suporte a crises de saúde mental para membros da comunidade que enfrentam a falta de moradia, indivíduos com problemas de saúde mental que trabalham como profissionais do sexo, com problemas de saúde mental e usuário de drogas, incluindo profissionais do sexo. A promoção da saúde para tais indivíduos é realizada por meio de oficinas, eventos sociais recreativos, o uso da terapia com animais e estratégias para redução do isolamento social, a consulta para hipertensão arterial e a consulta para idosos (ALL SAINTS, 2020).

Em um segundo momento foi realizado uma reunião com uma enfermeira do Foot Care Clinics (clínica de cuidados com os pés) que é um serviço onde uma Enfermeira da saúde da rua, especialista 
em cuidados com os pés, presta atendimentos voltados aos cuidados dos pés a população de rua (STREET HEALTH, 2020).

Pessoas em situação de rua têm um risco aumentado em comparação com a população em geral de desenvolver lesões relacionadas a queimaduras de gelo ou hipotermia que podem causar morbidade ou morte graves e representam um grave problema de saúde pública (NOE; JIN; WOLKIN, 2012 ). Um estudo realizou um levantamento de prontuários nos departamentos de emergência entre 2004 e 2015 em Toronto, Canadá. Os resultados apontaram 97 eventos hipotérmicos, sendo 79 lesões e 18 óbitos, o estudo também demonstra que a chances de ocorrência de um novo evento hipotérmico aumenta 1,64 vezes com cada decréscimo de $5^{\circ} \mathrm{C}$ na temperatura (ZHANG, 2019). Desta forma intervenções de saúde pública direcionadas são necessárias para reduzir os eventos hipotérmicos, dentre eles o risco de lesões relacionadas ao frio entre pessoas que sofrem com falta de moradia.

A foot care clinics é um dos serviços oferecidos pelo Street Heath, que é uma agência comunitária sem fins lucrativos que melhora a saúde de moradores de rua e sub-abrigados em Toronto (STREET HEALTH, 2020). A primeira clínica do Street Heath foi inaugurada em setembro de 1986, administrada por um grupo de enfermeiras voluntárias. Os serviços de saúde prestados são centrados no cliente e se caracterizam por serem, flexíveis, receptivos, baseados no modelo de redução de danos e multidisciplinar. O objetivo geral é o de avaliar as necessidades individuais de diferentes perspectivas e responder com soluções dinâmicas, visto que a população atendida tem como características a extrema pobreza, desemprego crônico, insegurança na moradia, má nutrição, alto estresse, uso de drogas e solidão (STREET HEALTH, 2020).

Em reunião com dois enfermeiros do Street Heath, recebemos materiais informativos sobre os diversos serviços em Toronto que ofereciam algum tipo de atendimento a população de rua e seus respectivos contatos. Uma segunda enfermeira com mais de 20 anos de experiência na cidade de Vancouver, nos informou que atualmente trabalha em Toronto no tratamento de feridas para pessoas vulneráveis e marginalizadas e que tem formação de mestrado voltado para a temática.

Outra reunião realizada foi com uma enfermeira do Health Bus Program uma unidade móvel que fornece um ponto de entrada para serviços de saúde para pessoas que frequentemente enfrentam barreiras no acesso aos serviços tradicionais de saúde.

Neste programa os serviços são prestados por uma enfermeira, conselheira de saúde mental, além de outros profissionais especializados. Os serviços incluem: tratamento e monitoramento de doenças temporárias ou crônicas, testes de DST e HIV, serviços de hepatite $C$, cuidados com os pés e feridas, cuidados preventivos e triagem (testes PAP, triagem do câncer colorretal), serviços de diabetes, educação e promoção da saúde, aconselhamento sobre dependência e redução de danos, apoio emocional e de crise, encaminhamento para apoios da comunidade, conexão com os serviços de atenção primária em andamento (SHERBORNE HEALTH, 2020). O programa também fornece 
suprimentos para redução de danos, educação sobre prevenção de overdose, incluindo distribuição de naloxona e suprimentos de saúde sexual seguros, como preservativos (SHERBORNE HEALTH, 2020).

Foram agendadas visitas a Foot Care Clinics e a Health Bus Program porém as mesmas foram inviabilizadas devido a pandemia de COVID 19. Assim outras atividades de extrema importância foram realizadas através da própria Ryerson University e demais comunidades científicas, com 16 webinar (total de 16 horas) que abordavam as temáticas voltadas para métodos de pesquisa, desigualdades sociais, enfrentamento ao coronavírus e tratamento de lesões.

A pesquisador principal realizou a anuência e se tornou-se membro de grupos em mídias sociais tais como Street Nurses Network, Toronto Drup-in Network, Street Health, Rise Up Movement, Nursing Students for Social Justice - Ryerson. Esses grupos possibilitaram o aprendizado da mesma através de outros enfermeiros membros acerca de como eles trabalham com as populações vulneráveis, bem como sobre advocacia política, a fim de garantir os direitos destas populações. Foi possível compreender que a advocacia é um pilar da Enfermagem e que para o desenvolvimento da profissão é necessário que os enfermeiros se envolvam mais em liderança, advocacia e formulação de políticas, pois somente desta forma poderemos ocupar espaço enquanto profissão e participar ativamente de tomadas de decisão, reformas e políticas de saúde e consequentemente garantir o direito da população a qual prestamos cuidados.

Terceira fase: Modificações do plano de trabalho

Decorrente a pandemia do COVID 19 em 23 de março de 2020 foi decretada pelo governo da província de Ontário o isolamento social e como consequência a suspensão de qualquer visita a locais de saúde, organizações não governamentais, organizações religiosas, eventos e reuniões presenciais. Assim o método de trabalho foi modificado, onde foram pactuados com 7 enfermeiros de quatro agências (Street Health; Public Health Nurse; Health Bus da Sherbourne Health; South Riverdale Community Health (entre) que aceitaram colaborar com o desenvolvimento desta etapa. 
Nesta fase foi criado um roteiro para que os enfermeiros consultados descrevessem aspectos principais de suas práticas com a população alvo. O roteiro contendo seis questões inspirou-se na metodologia científica do desenho da modelização qualitativa sistematizada proposto por Le Moigne (1990) que objetiva desenhar o modus operandi de um fenômeno complexo. A versão preliminar do roteiro foi revista por enfermeiro colaborador lotado no TPH quanto a sua clareza semântica ao transformar-se a linguagem metodológica em linguagem simples e compreensível para os enfermeiros da prática. Após revisão o roteiro foi enviado em um e-mail coletivo para 7 enfermeiras das agências que aceitaram colaborar dando as mesmas as duas opções para compartilhar as informações considerando a menor possibilidade de interferência na dinâmica dos trabalhos das mesmas. Sugeriu-se a resposta direta no e-mail ou a gravação de um áudio individual pelo telefone celular. As perguntas que guiaram esta fase da exploração do campo foram:

a) O que acontece quando a clientela precisa de cuidados de Enfermagem para lesões de pele?

b) Descreva o contexto em que esse tipo de assistência de Enfermagem é prestada.

c) Em que circunstâncias acontece o cuidado de Enfermagem?

d) Ao cuidar desta clientela, quais são os problemas mais comuns e suas consequências para você e para a clientela?

e) Que tipo de mudanças nos cuidados e procedimentos são guiados por protocolos de cuidados e / ou políticas?

f) Quais são os resultados usuais das mudanças implementadas e das intervenções gerais de Enfermagem?

Essa terceira fase se encontra em andamento, onde se obteve o retorno de alguns questionários. Com uma segunda rodada de e-mails para mais 4 enfermeiros, desde que os colaboradores que inicialmente se comprometeram a ajudar com as narrativas de ações, relataram uma dificuldade de fazê-lo, já que mais de um terço de suas equipes de suas clínicas encontravam-se em licença médica devido infecção de COVID 19. Tendo o ínicio de uma análise preliminar destes questionários, bem como se mantém aguardando o retorno dos demais questionários que subsidiará a construção de um modelo conceitual.

\section{Lições aprendidas}

A revisão da literatura, a experiência vivenciada na universidade Ryerson e o contato direto com enfermeiros atuantes em serviços de saúde, bem como os relatos recebidos na terceira fase, proporcionaram um conhecimento mais aprofundando sobre a característica da populacao em situação de rua de Toronto, bem como os programas de saúde existentes e a assistência prestada pelo 
enfermeiro a essa população. Tudo isso possibilitou uma construção de conhecimento por parte da doutoranda sobre a temática, dentro do contexto canadense.

Foi possível observar e analisar as diferenças existentes entre o perfil da população em situação de rua canadense e brasileira, suas especificidades, como o uso de drogas injetáveis e a crise do uso de opióides instaurada no país, bem como as políticas públicas voltadas para esta população elaboradas pelo governo canadense.

\section{Possiveis contribuições e transferência de conhecimentos para a prática da Enfermagem em Saúde Pública}

Acredita-se que este relato de experiência possa contribuir para ampliar o debate sobre o tema e possa ser expandir o conhecimento de profissionais de saúde, sobre as especificidades da assistência a essa população na cidade de Toronto. Disto pode resultar na transferência de conhecimento, aplicável a prática brasileira servindo de subsídios para a formulação de políticas públicas que possam atender as necessidades desta população brasileira.

Evidentemente demonstrar criticamente que, se torna necessário em todos os países que enfrentam tal problema de saúde pública, o distanciamento da adoção de estratégias que não abordem os determinantes sociais de saúde de pessoas em situações de vulnerabilidade. Ademais, deve-se rejeitar estratégias que embora inadvertidamente, resultem em pouco mais do que glorificar enfermeira(o)s que "cuidam dos pobres", bem como narrativas distorcidas da mídia sobre governos que pareçam ser progressistas no enfrentamento de tal problemática, mas com ações de baixa efetividade, perpetuando o problema existente.

\section{Limitações}

A limitação no estudo deve-se a interrupção do trabalho direto e a permanência nas clínicas e serviços devido a pandêmia. Tal indisponibilidade, impossibilitou o acompanhamento de interações diretas entre clientes e enfermeiros, provendo cuidados nas clínicas e nas ruas em Toronto.

\section{Conclusão}

Em suma, a experiência vivenciada no contexto profissional e social, promoveu o crescimento profissional, acadêmico e científico, além do desenvolvimento do pensamento crítico e conceitual sobre a implementação de políticas públicas de promoção da saúde e a reformulação do trabalho de Enfermagem com população em situação de rua e o desenvolvimento de conhecimentos que serão transferidos em um contexto de Enfermagem global. A experiência desta imersão que proporcionou uma interpretação de informações empíricas e um refinamento de habilidades de pensamento 
conceitual que podem ser transferidos para a realidade social brasileira. No futuro tal conhecimento poderá aumentar a visibilidade social dos resultados da pesquisa de doutoramento respondendo algumas lacunas de conhecimento principalmente no campo das políticas de saúde no Brasil para o tratamento de lesões periféricas em população em situação de rua.

Finalmente, a oportunidade de participação no ELAP produziu múltiplos dividendos relativos a conscientização da doutoranda em seu novo papel de líder social sobre a temática, bem como influenciadora de demais enfermeiros brasileiros, estudantes de graduação e pós graduação acerca da Enfermagem como uma profissão capaz de realizar um advocacia política e defender e assegurar os direitos das pessoas às quais prestam cuidados, em especial pessoas em situação de vulnerabilidade social. Bem como de participar ativamente na tomada de decisões, reformulações de políticas públicas e ações governamentais que gerem mudanças na saúde e nos cuidados a população.

\section{Agradecimentos}

Agradecemos a Enfa. Cristina Usanov (Ryerson University) e ao Enf. Mohamed Mohamed (Toronto Public Health) por facilitarem o processo de interligação da autora principal com a rede de enfermeiros que trabalham com população em situação de rua. A Enfa. Erin Telegdi (South Riverdale Community Health Centre), Enfa. Amanda Catching (Public Health Nurse), Enfa. Eileen Murphy (Health Bus da Sherbourne Health), Enfa. Liz Tevlin (Street Health), Enfa. Kelly White (Street Health), Enfa. Joyce Rankin (Street Health), Enfa. Elizabeth Harrison (Street Health) que compartilharam suas experiências e seus conhecimentos acerca da temática A Dra. Enfa. Corinne Hart (Ryerson University) e a Dra. Enfa. Denise Gastaldo (University of Toronto) pelo apoio técnico-científico. E aos membros da igreja All Saints Community Center-Church Toronto por me receberem em sua instituição e me apresentarem o trabalho que realizam para tal população. Conflitos de interesse: Os autores declaram não haver conflito de interesses. Os financiadores não tiveram nenhum papel no desenho do estudo; na redação do manuscrito, ou na decisão de publicar o artigo.

\section{Referências}

ALL SAINTS [homepage na internet]. Our Programs - Fulfilling a Great Need. 2020. Acesso em: 26 de maio de 2020. Disponível em: http://allsaintstoronto.com/our-programs/

BERGAMO S, PARISI G, JARRE P. "Harm reduction in Italy: the experience of an unsanctioned supervised injection facility run by drug users", Drugs and Alcohol Today, Vol. 19 No. 2, pp. 59-71; 2019. Acesso em: 24 de abril de 2020. Disponível em: https://doi.org/10.1108/DAT-03-2018-0011

CAMARGO, B. P. Vivência em Consultório na Rua do Rio de Janeiro: a situação de rua sob uma nova perspectiva. Revista Brasileira de Medicina de Família e Comunidade, v. 11, n. 38, p. 1-3, 15 ago. 2016. Acesso em: 24 de abril de 2020. Disponível em:

https://rbmfc.org.br/rbmfc/article/view/1269 
CANADIAN COUNCIL ON SOCIAL DETERMINANTS OF HEALTH. A Review of Frameworks on the Determinants of Health. ISBN: 978-0-9937151-6-7; 2015. Acesso em: 24 de abril de 2020. Disponivel em: http://ccsdh.ca/images/uploads/Frameworks_Report_English.pdf

DE TILIO, R; DE OLIVEIRA, J. Cuidados e atenção em saúde da população em situação de rua. Psicologia em Estudo, v. 21, n. 1, p. 101-113, 2016. Acesso em: 24 de abril de 2020. Disponível em: https://doi.org/10.4025/psicolestud.v21i1.27142

DOBERSTEIN, C. Building a Collaborative Advantage: Network Governance and Homelessness Policy-Making in Canada. UBC Press, Vancouver, 2016. Acesso em: 24 de abril de 2020. Disponível em: https://search.proquest.com/openview/a231800999456fff7ae7aff970f5fc10/1?pqorigsite $=$ gscholar $\& \mathrm{cbl}=35731$

ENGSTROM. E.M, et al. Equipe "Consultório na Rua" de Manguinhos, Rio de Janeiro, Brasil: práticas de cuidado e promoção da saúde em um território vulnerável. Ciência \& Saúde Coletiva [online]. 2016, v. 21, n. 6. Acesso em: 24 de abril de 2020. Disponível em:

https://www.scielosp.org/article/csc/2016.v21n6/1839-1848/pt/

FIORATI, R. C., CARRETTA, R. Y. D., KEBBE, L. M., CARDOSO, B. L., \& XAVIER, J. J. D. S. (2016). As rupturas sociais e o cotidiano de pessoas em situação de rua: estudo etnográfico. Revista Gaúcha de Enfermagem, 37(SPE). Acesso em: 25 de abril de 2020. Disponível em: http://dx.doi.org/10.159o/1983-1447.2016.esp.72861

FOTH, T., LANGE, J., \& SMITH, K. (2018). Nursing history as philosophy-towards a critical history of nursing. Nursing Philosophy, 19(3), e12210. Acesso em: 25 de abril de 2020. Disponível em: https://doi.org/10.1111/nup.12210

FOWLER PJ, HOVMAND PS, MARCAL KE, DAS S. Solving homelessness from a complex systems perspective: insights for prevention responses. Annual review of public health, 40, 465-486; 2019. Acesso em: 24 de abril de 2020. Disponível em: https://www.annualreviews.org/doi/abs/10.1146/annurev-publhealth-040617-013553

GADDIs A, KENNEDY M C, NOSOVA E, MILLOY M J, HAYASHI K, WOOD E, KERR T. Use of on-site detoxification services co-located with a supervised injection facility. Journal of substance abuse treatment, 82, 1-6; 2017. Acesso em: 24 de abril de 2020. Disponível em:

https://doi.org/10.1016/j.jsat.2017.08.003

GOMES, T. B.; VECCHIA, M. D. Estratégias de redução de danos no uso prejudicial de álcool e outras drogas: revisão de literatura. Ciência \& Saúde Coletiva, v. 23, n. 7, p. 2327-2338, jul. 2018. Acesso em: 24 de abril de 2020. Disponível em:

https://www.scielosp.org/article/csc/2018.v23n7/2327-2338

GOVERNMENT OF CANADA-EDUCANADÁ [homepage na internet]. Emerging Leaders in the Americas Program (ELAP) - .Background. 2020. Acesso em: 24 de abril de 2020. Disponível em: https://www.educanada.ca/scholarships-bourses/can/institutions/elap-pfla.aspx?lang=eng

HAMILTON, N. \& BHATTI, T (1996). Population health promotion: An Integrated Model of Population Health and Health Promotion. Government of Canada. 1996. Acesso em: 24 de abril de 2020. Disponivel em: https://www.canada.ca/en/public-health/services/healthpromotion/population-health/population-health-promotion-integrated-model-population-healthhealth-promotion/measures-of-success.html 
HARDILL, K. From the grey nuns to the streets: A critical history of outreach nursing in canada. Public Health Nursing, 24(1), 91-97. 2007. Acesso em: 24 de abril de 2020. doi:10.1111/j.1525$1446.2006 .00612 . x$

HARRIS M, GADERMANN A, NORENA M. et al. Residential moves and its association with substance use, healthcare needs, and acute care use among homeless and vulnerably housed persons in Canada. Int J Public Health 64, 399-409; 2019. Acesso em: 24 de abril de 2020. Disponivel em: https://doi.org/10.1007/s00038-018-1167-6

HASFORD J, NELSON G, WORTON S K, MACNAUGHTON E, MACLEOD T, PIAT M, GOERING P. Knowledge translation and implementation of housing first in Canada: A qualitative assessment of capacity building needs for an evidence-based program. Evaluation and program planning, 75, 1-9; 2019. Acesso em: 24 de abril de 2020. Disponível em: https://www.sciencedirect.com/science/article/abs/pii/S0149718918302052

HEALTH CANADA [homepage na internet]. Social determinants of health and health inequalities. 2019. Acesso em: 24 de abril de 2020. Disponivel em: https://www.canada.ca/en/publichealth/services/health-promotion/population-health/what-determines-health.html

HEALTH CANADA [homepage na internet]. Asylum claims by year - 2019. 2019. Acesso em: 24 de abril de 2020. Disponível em: https://www.canada.ca/en/immigration-refugeescitizenship/services/refugees/asylum-claims/asylum-claims-2019.html

HENRY B, DOSANI N, HUYNH L, AMIRAULT N. Palliative care as a public health issue: understanding disparities in access to palliative care for the homeless population living in Toronto, based on a policy analysis. Current oncology, 24(3), 187-19; 2017. Acesso em: 24 de abril de 2020. Disponível em: https://doi.org/10.3747/c0.24.3129

HERMAN, D., OPLER, L., FELIX, A., VALÊNCIA, E., R. WYATT, \& SUSSER, E. Intervenção crítica no tempo: Impacto nos sintomas psiquiátricos. Journal of Nervous and Mental Disease , 188 (3), 135140. 2000. Acesso em: 25 de abril de 2020. Disponível em:

https://journals.Iww.com/jonmd/Abstract/2000/03000/A_Critical_Time_Intervention_with_Mentally _lll.2.aspx

JAMES K., GODWIN A. \& EVAN C. The experiences of front-line service providers of Housing First programme delivery in three communities in Ontario, Canada. International Journal of Housing Policy, 17:3, 396-416, 2017. Acesso em: 25 de abril de 2020. DOI: 10.1080/14616718.2016.1248528

JONES, Kristine et al. Cost-effectiveness of critical time intervention to reduce homelessness among persons with mental illness. Psychiatric Services, v. 54, n. 6, p. 884-890, 2003. Acesso em: 25 de abril de 2020. Disponivel em: https://doi.org/10.1176/appi.ps.54.6.884

WOODHALL-MELNIK., JULIA R., JAMES R., DUNN. A systematic review of outcomes associated with participation in Housing First programs. Housing Studies, 31:3, 287-304, 2016. Acesso em: 25 de abril de 2020. DOI: 10.1080/02673037.2015.1080816

LATIMER E A, RABOUIN D, CAO Z, LY, A, POWELL G, AUBRY T, VELDHUIZEN S. Costs of services for homeless people with mental illness in 5 Canadian cities: a large prospective follow-up study. CMAJ open, 5(3), E576; 2017. Acesso em: 24 de abril de 2020. Disponível em: $10.9778 /$ cmajo.20170018 
LENNON, Mary Clare et al. Capturing intervention effects over time: Reanalysis of a critical time intervention for homeless mentally ill men. American Journal of Public Health, v. 95, n. 10, p. 17601766, 2005. Acesso em: 24 de abril de 2020. Disponível em:

https://ajph.aphapublications.org/doi/full/10.2105/AJPH.2005.064402

LENNON, Mary Clare et al. Ministério do Desenvolvimento Social e do Combate à Fome. Pesquisa nacional sobre a população em situação de rua. Meta/MDS, Brasília, DF, abr. 2008a. Disponível em: http://portalarquivos2.saude.gov.br/images/pdf/2015/agosto/14/sumario-executivo- poprua.pdf.

MYER A J, BELISLE L. HIGHS and LOWS. An Interrupted Time-Series Evaluation of the Impact of North America's Only Supervised Injection Facility on Crime. Journal of Drug Issues, 48(1), 36-49, 2018. Acesso em: 24 de abril de 2020. Disponível em: https://doi.org/10.1177/0022042617727513.

NARASIMHAN L, GOPIKUMAR V, JAYAKUMAR V. et al. Responsive mental health systems to address the poverty, homelessness and mental illness nexus: The Banyan experience from India. Int J Ment Health Syst 13, 54. 201. Acesso em: 24 de abril de 2020. Disponível em: https://doi.org/10.1186/s13033-019-0313-8

OPIOID USE AND RELATED ADVERSE EVENTS IN ONTARIO. Ontario Drug Policy Research Network. 2016. Acesso em: 24 de abril de 2020. Disponível em: http://odprn.ca/wpcontent/uploads/2016/11/ODPRN-Opioid-Use-and-Related-Adverse-Events-Nov-2016.pdf.

PANKRATZ C, NELSON G, MORRISON M. The Implementation of a Rent Assistance Program and Its Impacts on Recovery Outcomes for Individuals Experiencing Chronic Homelessness.

Canadian Journal of Community Mental Health, 37(1), 49-63; 2018. Acesso em: 24 de abril de 2020. Disponível em: https://doi.org/10.7870/cjcmh-2018-006

PANKRATZ C, NELSON G, MORRISON M. A quasi-experimental evaluation of rent assistance for individuals experiencing chronic homelessness. Journal of Community Psychology, 45(8), 10651079; 2017. Acesso em: 24 de abril de 2020. Disponível em: https://doi.org/10.1002/jcop.21911

PAULA HCD, DAHER DV, KOOPMANS FF, FARIA MGDA, BRANDÃO PS, SCORALICK GBF. A implantação do Consultório na Rua na perspectiva do cuidado em saúde. Revista Brasileira de Enfermagem, 71, 2843-2847; 2018. Acesso em: 24 de abril de 2020. Disponível em: https://doi.org/10.1590/0034-7167-2017-0616

PAULY, B. Close to the street: nursing practice with people marginalized by homelessness and substance use. Homelessness and health in Canada, 2014. Acesso em: 24 de abril de 2020.

Disponivel em: http://library.oapen.org/bitstream/id/ogae3086-daf8-4729-aco750a86127bcc5/515362.pdf\#page $=222$

PEACOCK A, LEUNG J, LARNEY S, COLLEDGE S, HICKMAN M, REHM J, ALI R. Global statistics on alcohol, tobacco and illicit drug use: 2017 status report. Addiction, 113(10), 1905-1926; 2018. Acesso em: 24 de abril de 2020. Disponível em: https://doi.org/10.1111/add.14234

POTTIE, $K_{1}$. et al. Clinical guideline for homeless and vulnerably housed people, and people with lived homelessness experience. CMAJ, v. 192, n. 10, p. E240-E254, 2020. Acesso em: 24 de abril de 2020. Disponível em: https://doi.org/10.1503/cmaj.190777 
PHILLIPS, S. D., BURNS, B. J., EDGAR, E. R., MUESER, K. T., LINKINS, K. W., ROSENHECK, R. A., MCDONEL HERR, E. C. Moving assertive community treatment into standard practice. Psychiatric Services, 52(6), 771-779. 2001. Acesso em: 24 de abril de 2020. DOI:10.1176/appi.ps.52.6.771

RAMSAY N, HOSSAIN R, MOORE M, MILO M, BROWN A. CUIDADOS DE SAÚDE EM SITUAÇÃO DE RUA: BARREIRAS, FACILITADORES E AS EXPERIÊNCIAS VIVIDAS DE PESSOAS EM SITUAÇÃO DE RUA QUE ACESSAM OS SERVIÇOS DE SAÚDE EM UM MUNICÍPIO DA REGIÃO CANADENSE. Pesquisa qualitativa em saúde , 29 (13), 1839-1849; 2019. Acesso em: 24 de abril de 2020. Disponível em: https://doi.org/10.1177/1049732319829434

REGISTERED NURSES' ASSOCIATION OF ONTARIO [homepage na internet]. NURSING BEST PRACTICE GUIDELINES. Acesso em: 24 de abril de 2020. Disponível em: https://rnao.ca/bpg

REZAEI F, NOROOZI A, ARMOON B, FARHOUDIAN A, MASSAH O, SHARIFI H, MANSOURIAN M. Social determinants and hepatitis $C$ among people who inject drugs in Kermanshah, Iran: Socioeconomic status, homelessness, and sufficient syringe coverage. Journal of Substance Use, 22(5), 474-478, 2017. Acesso em: 24 de abril de 2020. Disponível em:

https://www.tandfonline.com/doi/abs/10.1080/14659891.2016.1245793

SHERBORNE HEALTH [homepage na internet]. Health Bus Program. 2020. Acesso em: 26 de maio de 2020. Disponivel em:https://sherbourne.on.ca/primary-family-health-care/urban-health/healthbus/

STREET HEALTH [homepage na internet]. Health Care at Street Level. 2020. Acesso em: 26 de maio de 2020. Disponivel em: https://www.streethealth.ca/about\#.XsxbZGhKjIU

SOUZA, F. É.; RONZANI, T. M. Challenges to harm reduction practices in primary health care. Psicolestud. v. 23, 59-68, 2018. Acesso em: 24 de abril de 2020. Disponível em: https://www.researchgate.net/profile/Telmo-

Ronzani/publication/324919112_CHALLENGES_TO_HARM_REDUCTION_PRACTICES_IN_PRIMAR Y_HEALTH_CARE/links/5aeb281aa6fdcco3cdg10840/CHALLENGES-TO-HARM-REDUCTIONPRACTICES-IN-PRIMARY-HEALTH-CARE.pdf

SUSSER, E., et al. Preventing recurrent homelessness among mentally ill men: a" critical time" intervention after discharge from a shelter. American Journal of Public Health, v. 87, n. 2, p. 256262 , 1997. Acesso em: 24 de abril de 2020. Disponível em:

https://ajph.aphapublications.org/doi/abs/10.2105/AJPH.87.2.256

TORONTO PUBLIC HEALTH. Street Needs Assessment. Toronto; 2020. Acesso em: 08 de abril de 2020. Disponivel em: https://www.toronto.ca/community-people/health-wellness-care/healthprograms-advice/harm-reduction-supplies-and-locations/

TORONTO PUBLIC HEALTH. Supervised Injection Services. Toronto; 2020. Acesso em: 08 de abril de 2020. Disponivel em: https://www.toronto.ca/community-people/health-wellness-care/healthprograms-advice/supervised-injection-services/

UNITED NATIONS. Commission for Social Development. New York, ISSN 0251-9666, 2020. Acesso em: 24 de abril de 2020. Disponível em: https://undocs.org/en/E/2020/26

VIEIRA EW, GAZZINELLI A. Grau de integração da Atenção Primária à Saúde de município de pequeno porte na Rede de Atenção à Saúde. Saúde e Sociedade, 26, 448-461, 2017. Acesso em: 24 de abril de 2020. Disponível em: https://www.scielosp.org/article/sausoc/2017.v26n2/448-461/pt/ 
WORLD HEALTH ORGANIZATION. WHO Housing and health guidelines. ISBN : 9789241550376. (1)1-175, 2018. Acesso em: 24 de abril de 2020. Disponível em: https://apps.who.int/iris/bitstream/handle/10665/276001/9789241550376-eng.pdf?ua=1

WORTON S K, HASFORD J, MACNAUGHTON E, NELSON G, MACLEOD T, TSEMBERIS S, RICHTER $T$. Understanding systems change in early implementation of Housing First in Canadian communities: An examination of facilitators/barriers, training/technical assistance, and points of leverage. American journal of community psychology, 61(1-2), 118-130; 2018. Acesso em: 24 de abril de 2020. Disponível em: https://doi.org/10.1002/ajcp.12219

ZHANG, P., WIENS, K., WANG, R., LUONG, L., ANSARA, D., GOWER, S., ... \& HWANG, S. W. Cold Weather Conditions and Risk of Hypothermia Among People Experiencing Homelessness:

Implications for Prevention Strategies. International journal of environmental research and public health, 16(18), 3259; 2019. Acesso em: 24 de abril de 2020. Disponível em:

https://doi.org/10.3390/ijerph16183259.

\section{Detalhes dos autores}

Bianca Campos Oliveira

Doutoranda em Ciências do Cuidado em Saúde, Universidade Federal Fluminense. E-mail: bianca.campos.oliveira@gmail.com.

Margareth Santos Zanchetta

Professora Associada da Daphne Cockwell School of Nursing, Ryerson University, Toronto, Canada. E-mail: mzanchet@ryerson.ca.

Beatriz Guitton Renaud Baptista de Oliveira

Professora Titular da Universidade Federal Fluminense; Coordenadora do Programa de Pós Graduação em Ciências do Cuidado em Saúde, Universidade Federal Fluminense; Coordenadora da Área da Saúde - FAPERJ. E-mail: beatrizguitton@globo.com. 